\title{
Is the frozen elephant trunk technique justified for chronic type A aortic dissection in Marfan syndrome?
}

\author{
Yu Chen ${ }^{1 \#}$, Wei-Guo Ma ${ }^{1,2 \#}$, Jian-Rong Li ${ }^{1}$, Jun Zheng ${ }^{1,2}$, Yong-Min Liu ${ }^{1,2}$, Jun-Ming Zhu ${ }^{1,2}$, Li-Zhong Sun $^{1,2}$ \\ ${ }^{1}$ Department of Cardiovascular Surgery, Beijing Anzhen Hospital, Capital Medical University, and Beijing Institute of Heart Lung and Blood Vessel \\ Diseases, Beijing 100029, China; ${ }^{2} \mathrm{Fu}$ Wai Hospital and Cardiovascular Institute, Chinese Academy of Medical Sciences, Beijing 100037, China \\ \#These authors contributed equally to this work. \\ Correspondence to: Wei-Guo Ma, MD. Department of Cardiovascular Surgery, Beijing Anzhen Hospital, Beijing 100029, China. \\ Email:wgma@yahoo.com.
}

\begin{abstract}
Background: Chronic type A aortic dissection (cTAAD) in Marfan syndrome (MFS) is rare. Surgical experience is limited and the role of frozen elephant trunk (FET) technique remains undefined. We seek to evaluate the safety and efficacy of the total arch replacement (TAR) and FET technique for cTAAD in MFS. Methods: The clinical data of sixty-eight patients with MFS undergoing FET and TAR for cTAAD were analyzed.

Results: Mean age was 35.8 \pm 9.7 years and thirty-nine were male (57.4\%). Operative mortality was $10.3 \%$ (7/68). Stroke occurred in one (1.5\%), re-exploration for bleeding in five (7.3\%), low cardiac output in four $(5.9 \%)$, and acute renal failure in two $(2.9 \%)$. Follow-up was complete in $100 \%(61 / 61)$ at mean $7.3 \pm 4.0$ years. The false lumen was obliterated in $73.5 \%$ across FET and 50.0\% in unstented descending aorta (DAo). Distal dilation occurred in twenty patients, six of whom underwent thoracoabdominal aortic replacement, one abdominal aortic replacement and one thoracic endovascular aortic repair (TEVAR). Late death occurred in five. At ten years, 59.8\% were free from distal aortic dilation, and the incidences were $23.2 \%$ for death, $14.4 \%$ for distal reoperation, and $62.4 \%$ for reoperation-free survival. Predictors for operative mortality were extra-anatomic bypass [odds ratio (OR), 229.592; $\mathrm{P}=0.036$ ], preoperative maximal size (DMax) of aortic sinuses (mm) (OR, 1.134; $\mathrm{P}=0.032)$ and cardiopulmonary bypass $(\mathrm{CPB})$ time (minute) (OR, 1.061; $\mathrm{P}=0.041$ ). Risk factors for aortic dilatation included patent false lumen at diaphragmatic hiatus [hazard ratio (HR), 5.374; $\mathrm{P}=0.008$ ], preoperative $\mathrm{DMax}(\mathrm{mm})$ of proximal DAo $(\mathrm{HR}, 1.068 ; \mathrm{P}=0.001)$ and renal arteries (HR, 1.102; $\mathrm{P}=0.005)$ which also predicted distal reoperation (HR, 1.149; $\mathrm{P}=0.001)$. The time from onset to operation (day) (HR, 1.002; $\mathrm{P}=0.004)$ and $\mathrm{CPB}$ time (minute) (HR, 1.032; $\mathrm{P}=0.036)$ predicted late death.

Conclusions: This study shows that the TAR and FET technique is a safe and durable approach to cTAAD in patients with MFS. The operation should be performed as early as possible to optimize clinical outcomes.
\end{abstract}

Keywords: Aortic dissection; Marfan syndrome (MFS); frozen elephant trunk (FET); outcomes; survival; reoperation

Submitted Jan 28, 2020. Accepted for publication Mar 03, 2020.

doi: $10.21037 /$ acs.2020.03.10

View this article at: http://dx.doi.org/10.21037/acs.2020.03.10

\section{Introduction}

Although survivors of acute type A aortic dissection (TAAD) ultimately enter a chronic disease course $(1,2)$, they are still faced with high risk of life-threatening aortic complications, such as aortic rupture, patent false lumen, aneurysmal dilation and malperfusion syndromes, all of which necessitate reoperation $(3,4)$. However, scarce data have been published on the surgical outcomes of chronic type A aortic dissection (cTAAD) repair $(5,6)$. In particular, the role of total arch replacement (TAR) using the frozen elephant trunk (FET) technique remains unclear for 
cTAAD repair involving the aortic arch in patients with Marfan syndrome (MFS), who often have a narrowed true lumen compressed by a large patent false lumen (7).

Since 2003, we have been using the TAR and FET technique to repair TAAD for patients with MFS (8). Our experience has proven the benefits of the TAR and FET technique with respect to long-term survival and freedom from reoperation in such patients (7,9-12). In this study, we seek to assess the early and long-term outcomes of the TAR and FET technique for cTAAD in MFS, and to identify the risk factors for death and reoperation in such patients.

\section{Methods}

The Ethics Committees of Beijing Anzhen Hospital of Capital Medical University and Fu Wai Hospital of Chinese Academy of Medical Sciences approved this retrospective study.

\section{Patients}

Between September 2003 and October 2016, we performed TAR and FET for cTAAD involving the aortic arch or proximal descending aorta (DAo) in sixty-eight consecutive patients with MFS. The diagnosis of MFS was based on the Ghent and/or revised Ghent criteria $(13,14)$. According to the 2014 European Society of Cardiology guidelines, aortic dissection is divided into sub-acute (15-90 days) and chronic ( $>90$ days) (1). Patients with cTAAD who had a history of proximal aortic surgery were excluded from this study $(10,15)$. The diagnosis of cTAAD was confirmed by computed tomographic angiogram (CTA) in all patients. Malperfusion was diagnosed based on clinical symptoms and imaging evidence.

\section{Surgical indications and techniques}

In MFS patients with cTAAD, we would perform TAR and FET for the following pathologies: (I) an intimal tear located in the transverse arch or DAo; (II) aneurysm of the aortic arch or proximal DAo (>40 $\mathrm{mm}$ in diameter); (III) dissection, aneurysm, or occlusion of the brachiocephalic arteries. For cTAADs confined to the ascending aorta (i.e., DeBakey type II), we would choose an ascending aortic replacement if the arch and DAo are intact, or an ascending aortic and hemiarch replacement if the proximal arch is involved.

Our surgical technique of TAR and FET (the Sun operation) has been described in detail previously $(7,8,16-18)$. We routinely use unilateral selective antegrade cerebral perfusion through right axillary cannulation and moderate hypothermic circulatory arrest at $20-25{ }^{\circ} \mathrm{C}$. The procedure involves deployment of an FET, Cronus ${ }^{\circledR}$ (MicroPort, Shanghai, China) in the DAo, followed by TAR with a 4-branched vascular graft (Maquet Cardiovascular, Rastatt, Germany). To minimize the time of cerebral, myocardial and spinal cord ischemia, distal reperfusion is initiated once the distal anastomosis is completed. The left carotid artery is reconstructed first (after which rewarming is started and the brain is perfused bilaterally), followed by the ascending aorta (to resume myocardial perfusion), then the left subclavian artery, and finally, the innominate artery. In selected patients, the arch branches were reconstructed using a modified en bloc technique $(17,18)$. Near-infrared spectroscopy, bispectral index, and cerebrospinal fluid drainage are used in high-risk patients.

\section{Clinical and imaging follow-up}

CTA of the entire aorta was performed at one month (or discharge), six months, one year and annually henceforth to assess the maximal aortic size (DMax) and complications (endoleak, migration, etc.). The aortic diameter ( $\mathrm{mm}$ ) was measured from the outer contours of the aortic wall in the axial plane, and growth rate calculated by dividing the diameter difference between the first and last CTAs. CTA measurements and analyses were performed at four aortic levels: the stented proximal descending aorta (FET), unstented DAo, diaphragmatic hiatus $(\mathrm{DH})$ and renal arteries (RA). The statuses of the false lumen were classified as: completely thrombosed (obliterated) if no flow was present; partially thrombosed if both flow and thrombus were present; and patent if flow was present in the absence of thrombus.

Open or endovascular reintervention on the distal aorta was indicated for patients with a distal DMax of $>50 \mathrm{~mm}$. Earlier reintervention is recommended for patients with a positive family history $(19,20)$, or distal new entry $(21,22)$, or new onset of chest or back pain.

\section{Study endpoints}

The primary clinical endpoints included operative mortality, late death, dilation and distal aortic reoperations. Late death referred to all-cause mortality during followup, excluding operative mortality (defined as deaths within 
30 days of surgery or before final hospital discharge, including transfers). Distal aortic reoperation included any open or endovascular reintervention on the distal aorta.

Aortic dilatation was defined as a maximal diameter of $>50 \mathrm{~mm}$ (45 mm in patients with a family history of aortic surgery or rupture) or an average growth rate of $>5 \mathrm{~mm}$ /year at any aortic segment as detected by CTA during follow-up.

\section{Statistical analysis}

Statistical analysis was performed using SPSS for Windows 22.0 (IBM SPSS, Armonk, NY, USA) and Prism 8.3 for Mac (GraphPad, La Jolla, CA, USA). Data are expressed as the mean \pm standard deviation (SD) or number (percentage) and were compared using the Student $t$-test or MannWhitney $U$ for continuous variables, and the Pearson $\chi^{2}$ text or Fisher's exact for categorical variables, as appropriate. Risk factors for operative mortality and complications were identified with logistic regression. Predictors for distal aortic dilatation (binary), reoperation and late death were identified with Cox regression models. Survival and freedom from aortic dilatation and reoperation were estimated using the Kaplan-Meier method, and intergroup comparisons were made with the log-rank test. Competing risks analysis of death and reoperation were based on the decomposition of the hazard function into multiple, additive overlapping phases of hazard, as described by Blackstone et al. (23). All tests were 2 -sided and a $\mathrm{P}$ value of $<0.05$ was considered statistically significant.

\section{Results}

\section{Baseline characteristics}

Mean age of patients was $35.8 \pm 9.7$ (range, 19-57) years and thirty-nine were male $(57.4 \%)$. TAAD was sub-acute (15-90 days) in thirty-eight (55.9\%) and chronic (>90 days) in thirty $(44.1 \%)$.

The interval from onset of symptoms to diagnosis was 150 days on average (median 6.5 days, range 1 day to 7 years). The mean interval from symptomatic onset to operation was 306 days (median 64 days, range 15 days to 9 years), which was significantly longer in the chronic group compared to the sub-acute group $(643 \pm 791$ vs. $39 \pm 18$ days, $\mathrm{P}<0.001)$.

The majority of patients (94.1\%) sustained an acute onset of pain. Abrupt onset of severe chest pain was seen in $51.5 \%(35 / 68)$, and isolated back or abdominal pain in
$14.7 \%(10 / 68)$ during the acute course. Other symptoms included chest tightness accompanied by chest pain in $38.2 \%$ (26/68), back pain in 35.3\% (24/68), and orthopnea and lower extremity edema in $23.5 \%(16 / 68)$. Twenty patients $(29.4 \%)$ reported that the pain was "slight" or "transient". In eleven symptomatic patients (16.2\%), the dissection was misdiagnosed at first as myocardial infarction, lumbar muscle strain, gastroenteritis, pancreatitis, or pleurisy. In four asymptomatic patients (5.9\%), dissection was diagnosed incidentally during medical examination for other reasons.

The distal extent of aortic dissection was to the diaphragmatic hiatus in sixteen $(23.5 \%)$, to the renal arteries in seven $(10.3 \%)$ and to the iliac arteries in 45 patients $(66.2 \%)$. The arch vessels were involved in 68 patients $(100 \%)$, including left carotid artery in $61(89.7 \%)$ and innominate artery in $66(97.1 \%)$ and left subclavian artery in $60(88.2 \%)$.

Before surgery, the DMax at the levels of the aortic sinuses, arch, proximal descending aorta, diaphragmatic hiatus and renal arteries were $67.8 \pm 13.7,33.5 \pm 6.7$, $37.5 \pm 10.6,29.4 \pm 7.8$ and $25.6 \pm 6.8 \mathrm{~mm}$, respectively.

Preoperatively, malperfusion syndrome was diagnosed in six patients (two in sub-acute phase), including ischemia of the kidney in four cases, brain in one, and coronary artery in one, and acute heart failure secondary to severe aortic insufficiency in three (Table 1).

\section{Operative data}

The entry tear was located in the ascending aorta in sixtysix patients $(97.1 \%)$. An arch entry tear was found in two patients $(2.9 \%)$.

The times of cardiopulmonary bypass (CPB), cross-clamp and selective unilateral antegrade cerebral perfusion were $185.3 \pm 48.5,104.0 \pm 33.6,23.4 \pm 7.9$ minutes, respectively. The mean cooling nasopharyngeal temperature was $21.1 \pm 3.0^{\circ} \mathrm{C}$ (range, $15-25^{\circ} \mathrm{C}$ ). Bentall procedure with a mechanical valve was performed in sixty-two patients $(91.2 \%)$ and ascending aortic replacement in six (8.8\%). Coronary artery bypass grafting was performed in four (5.9\%) and mitral valve surgery in five patients (7.4\%).

An extra-anatomic bypass was performed in seven patients $(10.3 \%)$, six as a bailout, including ascending aorticto-femoral bypass in six and femoro-femoral bypass in one. All seven patients had a small true lumen and visceral or lower limb ischemia and were found to have a pressure difference of $>50 \mathrm{mmHg}$ between the upper and lower 


\begin{tabular}{|c|c|c|c|c|}
\hline Variable & Total $(n=68)$ & Subacute $(n=38)$ & Chronic $(n=30)$ & $P$ value \\
\hline Age, y & $35.8 \pm 9.7$ & $36.4 \pm 10.8$ & $35.1 \pm 8.3$ & 0.578 \\
\hline Male gender & 39 (57.4\%) & $24(63.2 \%)$ & $15(50.0 \%)$ & 0.276 \\
\hline Hypertension & $22(32.4 \%)$ & $10(26.3 \%)$ & $12(40.0 \%)$ & 0.231 \\
\hline Time from onset to surgery (day) & $305.5 \pm 601.7$ & $39.1 \pm 17.7$ & $642.9 \pm 790.8$ & $<0.001$ \\
\hline Median (interquartile range) & $63.5(31.3,356)$ & $32.5(25.5,50)$ & $372.0(241.5,732)$ & \\
\hline \multicolumn{5}{|l|}{ Clinical manifestation } \\
\hline Abrupt severe chest pain & $35(51.5 \%)$ & $24(63.2 \%)$ & $11(36.7 \%)$ & 0.030 \\
\hline Weakness & $1(1.5 \%)$ & $1(2.6 \%)$ & 0 & 0.371 \\
\hline Asymptomatic & $4(5.9 \%)$ & 0 & $4(13.3 \%)$ & 0.020 \\
\hline \multicolumn{5}{|l|}{ Preoperative aortic diameter, $\mathrm{mm}$} \\
\hline Aortic sinus & $67.8 \pm 13.7$ & $66.6 \pm 13.7$ & $69.4 \pm 13.8$ & 0.416 \\
\hline Aortic arch & $33.5 \pm 6.7$ & $33.4 \pm 6.6$ & $33.6 \pm 6.9$ & 0.936 \\
\hline Proximal descending aorta & $37.5 \pm 10.6$ & $35.4 \pm 9.4$ & $40.1 \pm 11.7$ & 0.111 \\
\hline Mid-descending aorta & $30.1 \pm 7.4$ & $29.2 \pm 6.4$ & $31.2 \pm 8.5$ & 0.339 \\
\hline Malperfusion syndrome* & $6(8.8 \%)$ & $2(5.3 \%)$ & $4(13.3 \%)$ & 0.244 \\
\hline Stroke ${ }^{*}$ & $1(1.5 \%)$ & 0 & $1(3.3 \%)$ & 0.257 \\
\hline Acute myocardial infarction & $1(1.5 \%)$ & $1(2.6 \%)$ & 0 & 0.371 \\
\hline Acute kidney injury & $4(5.9 \%)$ & $1(2.6 \%)$ & $3(10.0 \%)$ & 0.200 \\
\hline Heart failure & $3(4.4 \%)$ & $1(2.6 \%)$ & $2(6.7 \%)$ & 0.421 \\
\hline \multicolumn{5}{|l|}{ Arch vessel involvement } \\
\hline Innominate artery & $66(97.1 \%)$ & 36 (94.7\%) & $30(100 \%)$ & 0.202 \\
\hline Left carotid artery & $61(89.7 \%)$ & $32(84.2 \%)$ & $29(96.7 \%)$ & 0.093 \\
\hline Left subclavian artery & $60(88.2 \%)$ & $34(89.5 \%)$ & $26(86.7 \%)$ & 0.721 \\
\hline
\end{tabular}




\begin{tabular}{|c|c|c|c|c|}
\hline Variable & Total $(n=68)$ & Subacute $(n=38)$ & Chronic $(n=30)$ & $P$ value \\
\hline \multicolumn{5}{|l|}{ Operative data } \\
\hline Cardiopulmonary bypass time, min & $185.3 \pm 48.5$ & $189.4 \pm 49.3$ & $180.1 \pm 47.8$ & 0.437 \\
\hline Cross-clamp time, $\min$ & $104.0 \pm 33.6$ & $105.9 \pm 37.3$ & $101.5 \pm 28.8$ & 0.595 \\
\hline \multicolumn{5}{|l|}{ Concomitant procedures } \\
\hline Composite graft root replacement & $62(91.2 \%)$ & $34(89.5 \%)$ & $28(93.3 \%)$ & 0.577 \\
\hline Extra-anatomic bypass & $7(10.3 \%)$ & $3(7.9 \%)$ & $4(13.3 \%)$ & 0.464 \\
\hline Ascending aortic replacement & $6(8.8 \%)$ & $4(10.5 \%)$ & $2(6.7 \%)$ & 0.577 \\
\hline FET diameter (mm) & $26.0 \pm 1.5$ & $25.7 \pm 1.4$ & $26.5 \pm 1.5$ & 0.020 \\
\hline FET length $(\mathrm{mm})$ & $100.3 \pm 4.2$ & $100.0 \pm 4.6$ & $100.7 \pm 3.7$ & 0.522 \\
\hline \multicolumn{5}{|l|}{ Operative outcomes } \\
\hline Mortality & $7(10.3 \%)$ & $3(7.9 \%)$ & $4(13.3 \%)$ & 0.464 \\
\hline Morbidity & $19(27.9 \%)$ & $9(23.7 \%)$ & $10(33.3 \%)$ & 0.379 \\
\hline Stroke & $1(1.5 \%)$ & $1(2.6 \%)$ & 0 & 0.371 \\
\hline Low cardiac output & $4(5.9 \%)$ & $1(2.6 \%)$ & $3(10.0 \%)$ & 0.200 \\
\hline Acute renal failure & $2(2.9 \%)$ & $1(2.6 \%)$ & 1 (3.3\%) & 0.865 \\
\hline
\end{tabular}

limbs after implantation of the FET. Additional bailout procedures included lower-extremity arterial endovascular stenting and thoracoabdominal aortic replacement (TAAAR) in one patient each (Table 2).

\section{Operative mortality and morbidity}

The operative mortality rate was $10.3 \%$ (7/68). The most common cause of death was multiorgan failure in five patients (7.4\%). Among these, three underwent an ascending aortic-to-femoral bypass and one received a femoro-femoral bypass. Left ventricular rupture after mitral valve replacement and distal aortic rupture led to one death each (1.5\%).

Operative complications included stroke in one patient $(1.5 \%)$, acute renal failure in two $(2.9 \%)$, low cardiac output in four $(5.9 \%)$, and re-exploration for bleeding in five patients (7.4\%). No spinal cord injury occurred.

\section{Long-term outcomes}

Clinical and radiological follow-up was complete in $100 \%$ (61/61) for a mean duration of $7.3 \pm 4.0$ (median, 7.2; range, $0.3-14.8$ ) years, extending to 5 years in $87 \%$ and beyond 10 years in $76 \%$.

\section{Thrombosis of false lumen}

Before discharge, complete thrombosis (obliteration) of the false lumen occurred at the proximal end of the FET in $96.7 \%$ of patients (58/60) and type I endoleak was detected in two patients. At the distal end of FET, unstented mid-DAo and diaphragmatic hiatus, the false lumen 


\begin{tabular}{|c|c|c|c|c|c|c|c|}
\hline Aortic segment & Complete & Partial & Patent & Complete & Partial & Patent & $P$ value \\
\hline Proximal end of FET & 94.1 & 2.9 & 2.9 & 100.0 & 0 & 0 & 0.453 \\
\hline Distal end of FET & 73.5 & 20.6 & 5.9 & 88.5 & 11.5 & 0 & 0.264 \\
\hline Diaphragmatic hiatus & 35.3 & 20.6 & 44.1 & 26.9 & 19.2 & 53.8 & 0.730 \\
\hline
\end{tabular}

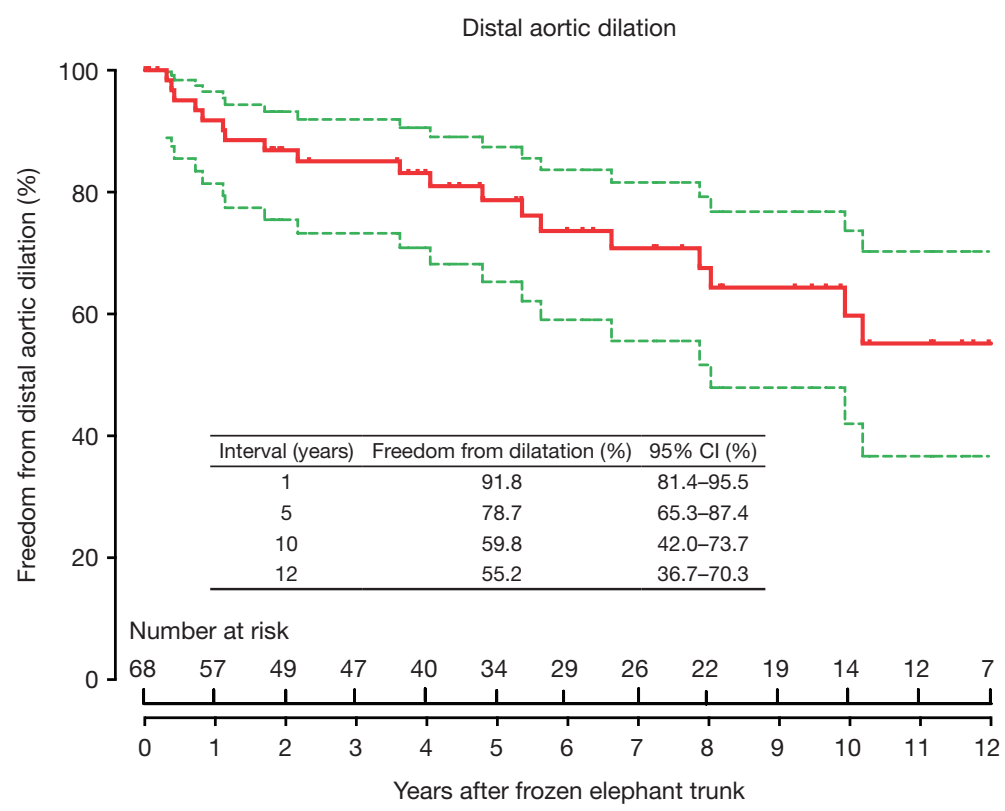

Figure 1 Freedom from distal aortic dilation.

was obliterated in $80.0 \%, 48.3 \%$ and $31.7 \%$; partially thrombosed in $16.7 \%, 21.7 \%$, and $20.0 \%$; and patent in $3.3 \%, 30.0 \%$, and $48.3 \%$, respectively (Table 3).

\section{Distal aortic dilatation}

The mean DMax at the levels of the FET, unstented midDAo, diaphragmatic hiatus and renal arteries were 39.8 , $34.8,35.2$ and $31.0 \mathrm{~mm}$ on the latest computed tomographic (CT) scan, as compared to 37.5, 30.1, 29.4 and $25.6 \mathrm{~mm}$ before surgery, respectively.

Distal aortic dilation occurred in twenty patients, including twelve in the sub-acute group (34.3\%) and eight in the chronic group $(30.8 \% ; \mathrm{P}=0.772)$. Among these, six were managed with open TAAAR, one with abdominal aortic replacement, one with thoracic endovascular aortic repair (TEVAR), three died of distal aortic rupture, eight were awaiting surgical repair and one with a distal DMax of $<45 \mathrm{~mm}$ was closely followed up.

The freedom from distal aortic dilatation was $91.8 \%$ (95\% confidence interval, CI, 81.4-95.5\%), 78.7\% (95\% CI, $65.3-87.4 \%$ ) and $59.8 \%$ (95\% CI, $42.0-73.7 \%)$ at 1,5 and 10 years, respectively (Figure 1).

\section{Late reintervention}

Reintervention on the dilated distal aorta included open TAAAR in six patients, abdominal aortic replacement in one patient, and TEVAR in one patient with a giant false lumen (Table 4). 


\begin{tabular}{|c|c|c|c|c|}
\hline Late death & $5(8.2 \%)$ & $1(2.9 \%)$ & $4(15.4 \%)$ & 0.078 \\
\hline Distal aortic rupture & $3(4.9 \%)$ & $1(2.9 \%)$ & $2(7.7 \%)$ & 0.388 \\
\hline Congestive heart failure and arrhythmia & $1(1.6 \%)$ & 0 & $1(3.8 \%)$ & 0.242 \\
\hline \multicolumn{5}{|l|}{ Late adverse events } \\
\hline Type la endoleak & $2(3.3 \%)$ & $2(5.7 \%)$ & 0 & 0.215 \\
\hline Leakage of coronary button & $2(3.3 \%)$ & $1(2.9 \%)$ & $1(3.8 \%)$ & 0.830 \\
\hline Dilatation of distal aorta & $20(32.8 \%)$ & $12(34.3 \%)$ & $8(30.8 \%)$ & 0.772 \\
\hline Thoracic endovascular aortic repair & $1(1.6 \%)$ & 0 & $1(3.8 \%)$ & 0.242 \\
\hline \multicolumn{5}{|l|}{ Maximal aortic diameter, $\mathrm{mm}$} \\
\hline Proximal descending aorta & $39.8 \pm 14.3$ & $38.7 \pm 12.3$ & $41.1 \pm 16.4$ & 0.562 \\
\hline Mid-descending aorta & $34.8 \pm 11.5$ & $34.8 \pm 11.6$ & $34.7 \pm 12.1$ & 0.976 \\
\hline Diaphragmatic hiatus & $35.2 \pm 11.5$ & $36.7 \pm 12.4$ & $33.4 \pm 10.3$ & 0.312 \\
\hline Renal arteries & $31.0 \pm 10.4$ & $31.6 \pm 11.3$ & $30.2 \pm 9.4$ & 0.632 \\
\hline
\end{tabular}

Discharge CTA showed that the distal end of FET entered the false lumen in four patients. The distal aorta remained undilated in one and eventually became dilated in three, which were managed with TAAAR.

The DMax of distal aorta at the time of distal reintervention was $58.0 \pm 17.1 \mathrm{~mm}$ (range, $42-90 \mathrm{~cm}$ ). After reintervention, eight patients were alive at mean follow-up of 4.0 \pm 2.7 years (range, $0.1-8.1$ years).

The freedom from distal reoperation was $96.6 \%(95 \%$ CI, $87.1-99.1 \%$ ), $90.2 \%$ (95\% CI, $77.7-95.9 \%$ ) and $82.0 \%$ (95\% CI, 66.7-90.8\%) at 1, 5 and 10 years, respectively (Figure 2).

Two patients undergoing Bentall procedure were found to have coronary button leak, which was successfully managed with open repair. The two patients with type Ia endoleak elected to have conservative management and are being closely monitored.

\section{Late survival}

Late death occurred in five patients. Three died of distal aortic rupture, one due to chronic heart failure and arrhythmia and one for non-cardiac causes.
Survival was $86.8 \%$ (95\% CI, 76.1-92.9\%), 86.8\% (95\% CI, $76.1-92.9 \%$ ), and $76.0 \%$ (95\% CI, $59.0-86.7 \%)$ at 1 , 5 and 10 years, respectively (Figure 3), which did not differ significantly between patients in the sub-acute and chronic groups $(\mathrm{P}=0.107)$ (Figure 4).

In competing outcomes analysis, the incidences were $13.2 \%$ and $23.2 \%$ for death, $9.2 \%$ and $14.4 \%$ for reoperation, and $77.6 \%$ and $62.4 \%$ were alive without distal reoperation, at 8 and 10 years, respectively (Figure 5).

\section{Risk factors for death, aortic dilation and distal reoperation}

Logistic regression identified that the risk factors for operative mortality were extra-anatomic bypass [odds ratio (OR), 229.592; 95\% confidence interval (CI), 1.42936,880.03; $\mathrm{P}=0.036$ ]; preoperative $\mathrm{DMax}$ of the aortic sinuses (OR 1.134; 95\% CI 1.011-1.271; $\mathrm{P}=0.032$ ) and time of CPB (in minutes) (OR, 1.061; 95\% CI, 1.003-1.123; $\mathrm{P}=0.041$ ).

In Cox regression, predictors for distal aortic dilatation were the preoperative maximal aortic diameter (in $\mathrm{mm}$ ) 


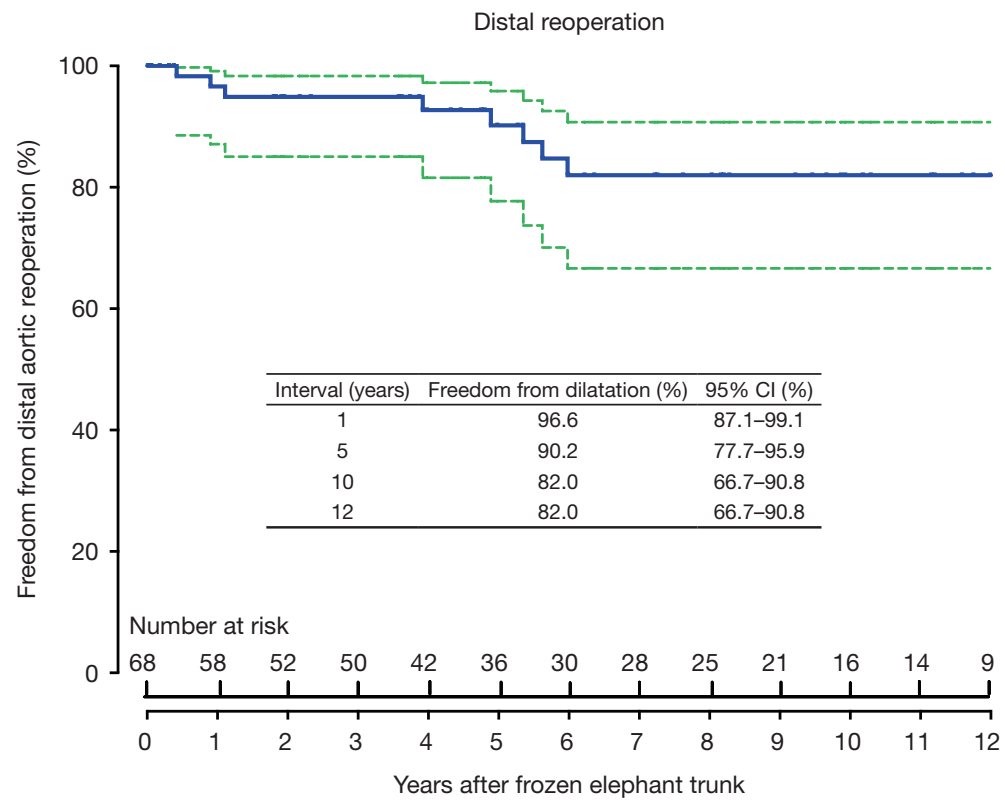

Figure 2 Freedom from distal aortic reoperation.

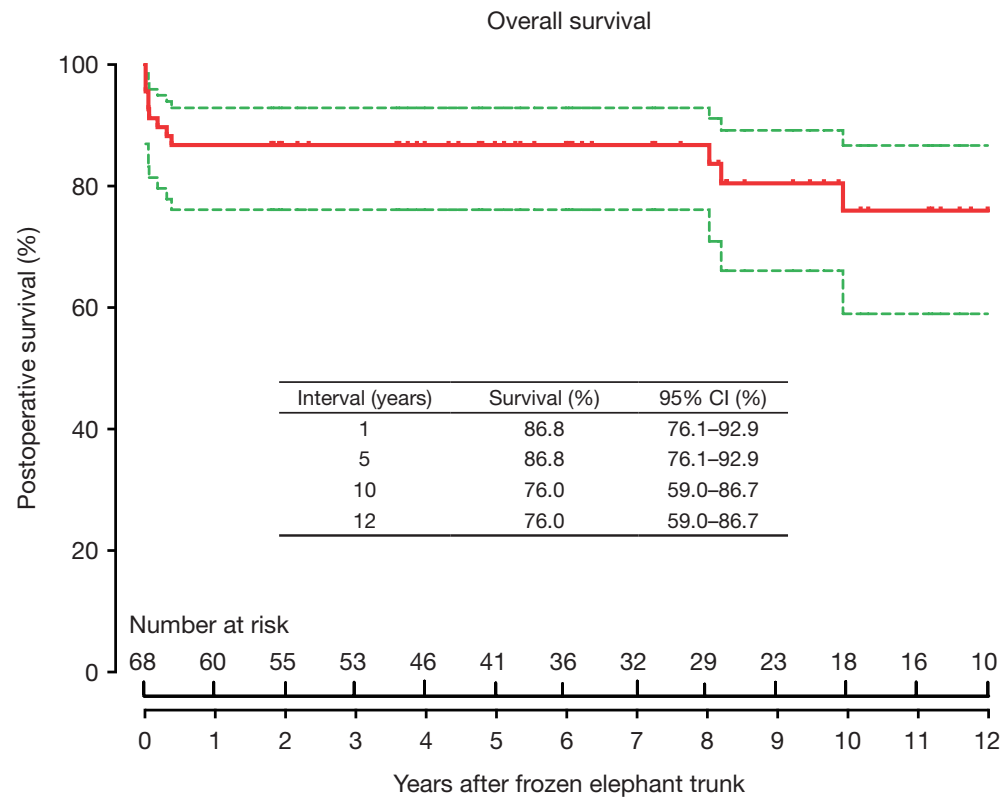

Figure 3 Overall survival.

of the proximal DAo (hazard ratio, HR, 1.068; 95\% CI, $1.026-1.112 ; \mathrm{P}=0.001)$ and at the renal arteries (HR, 1.102; 95\% CI, 1.030-1.178; P=0.005), and patent false lumen at the diaphragmatic hiatus (HR, 5.374; 95\% CI, $1.537-$ 18.788; $\mathrm{P}=0.008)$.

The maximal aortic size at the renal arteries $(\mathrm{mm})$ was predictive of late reoperation (HR, 1.149; 95\% CI, 1.059 1.247; $\mathrm{P}=0.001)$.

The risk factors for late death were the duration from symptom onset to operation (in days) (HR, 1.002; 95\% CI, 1.001-1.003; $\mathrm{P}=0.004)$, and the time of $\mathrm{CPB}$ (in minutes) (HR, 1.032; 95\% CI, 1.002-1.063; P=0.036) (Table 5). 


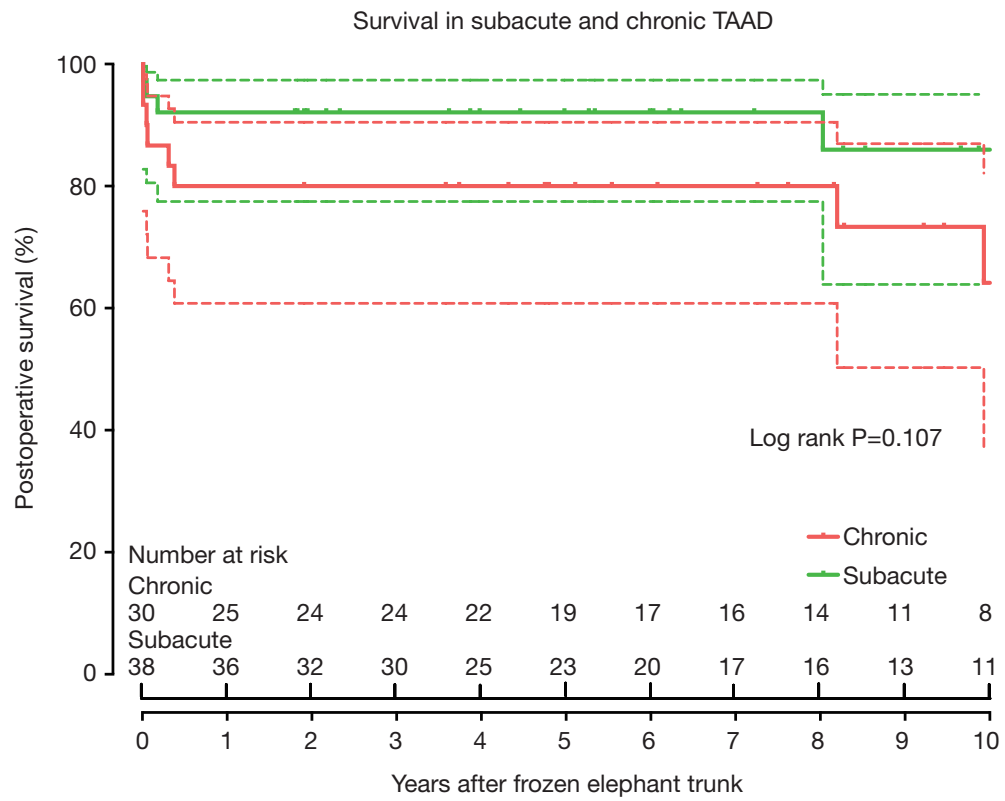

Figure 4 Survival in patients with subacute and chronic type A aortic dissection (TAAD).

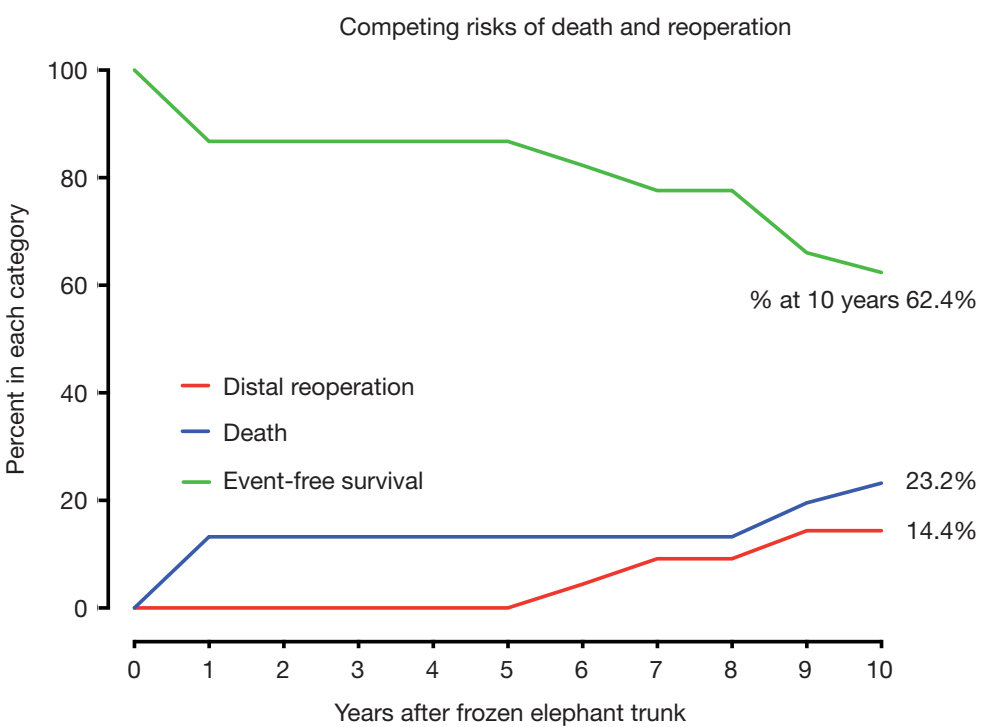

Figure 5 Competing risks of death and distal reoperation.

\section{Discussion}

While the majority of patients with TAAD are treated surgically upon presentation (24), only a small proportion of such patients experience delayed diagnosis and treatment. Therefore, cTAADs remain a rare and highly elusive entity, and, consequently, clinical experience is limited (2), especially in patients with connective tissue disorders.
In this series, $94.1 \%$ of patients were undiagnosed and untreated during the acute phase and survived despite the lack of therapy. Possible reasons include lack of or atypical symptoms, limited extent of dissection, and initial admission at a local nontertiary hospital without an aortic team (25). TAAD was an occasional finding in $5.9 \%$ and the pain was either "slight" or "transient" in $29.4 \%$ of patients, which suggests that patients with MFS may be more likely to 


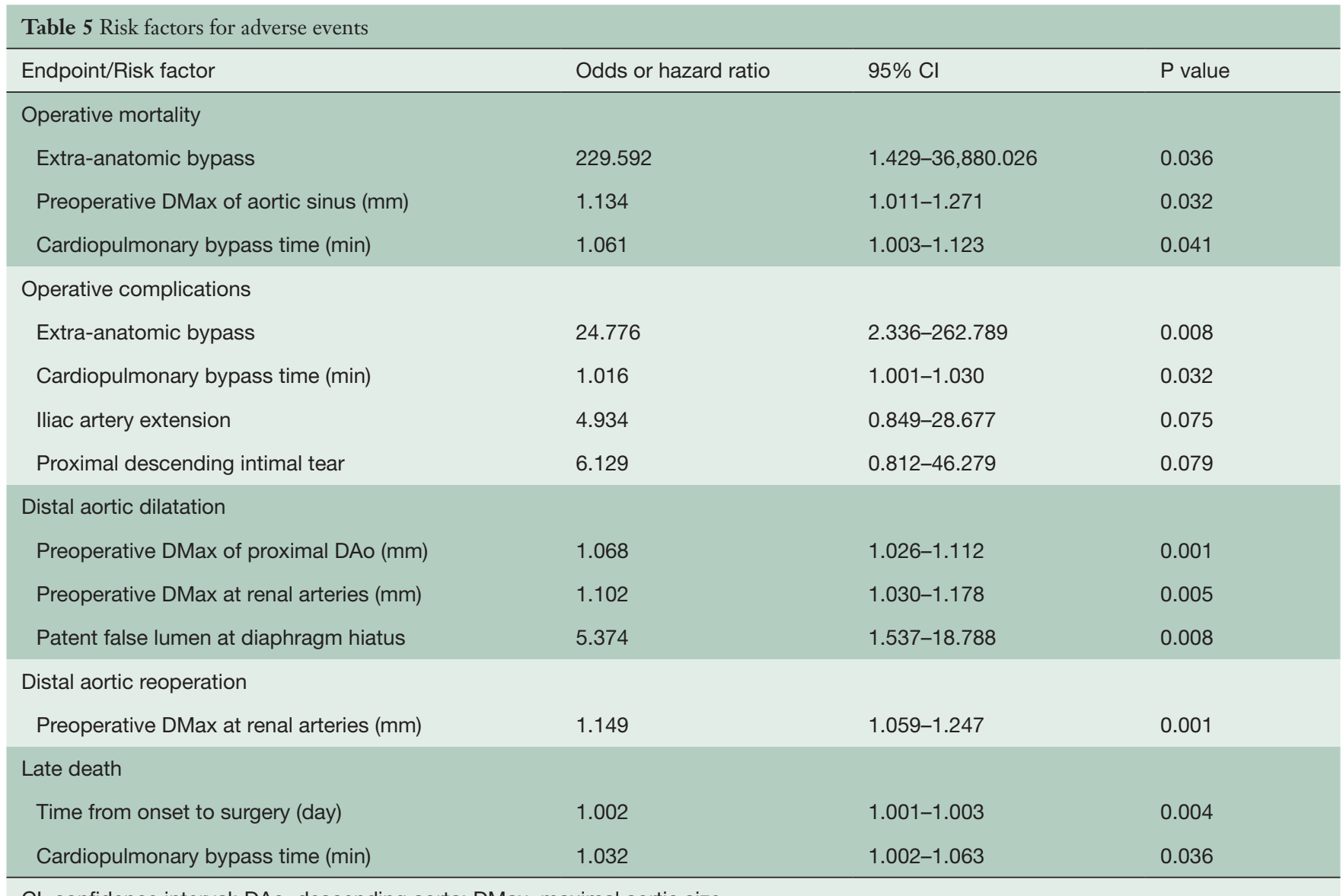

$\mathrm{Cl}$, confidence interval; DAo, descending aorta; DMax, maximal aortic size.

present with less severe or no pain (26).

Morphologically, chronic aortic dissections are characterized by dilation of the aorta, continued false lumen patency, thickening and immobility of the intimal flap, increased fibrosis in the media and deceleration of remodeling over time (2). Clinical experience with cTAAD is limited to a few studies (3-5) and current guidelines recommend surgery for patients with cTAAD when the ascending aortic diameter exceeds $55 \mathrm{~mm}$ (1). However, the optimal surgical procedure for cTAADs involving the distal arch or DAo and the timing of repair remain unclear in patients with MFS (27).

The thickened and stiffened dissection flap, and the small true lumen compressed by the false lumen in cTAAD increased the technical complexity and mortality risk of surgical repair (12). In this context, the FET technique is particularly well-suited for covering the proximal entry tear and alleviating or delaying distal aortic dilation $(28,29)$. In this series, operative mortality was $10.3 \%$, stroke rate
$1.5 \%$, and no spinal cord injury occurred; at 10 years, $60 \%$ were free from distal aortic dilation, while the cumulative incidences were $23.2 \%$ for death, $14.4 \%$ for distal reoperation, and $62.4 \%$ were alive without reoperation. These favorable results demonstrate the safety and efficacy of the FET technique for cTAAD in MFS, providing evidence for use of this extensive approach in such patients.

Nevertheless, an extra-anatomic bypass was found to increase the mortality risk drastically (OR, 229.6), leading to four among seven operative deaths in this series. Preoperative CT scan showed a narrowed true lumen compressed by an enlarged false lumen in all these patients, who also had a dilated thoracoabdominal aorta and sustained lower extremity or visceral ischemia. As a result, extra-anatomic bypass, endovascular stenting or TAAAR was necessitated as a bailout procedure, which markedly increased the risk for mortality and morbidity. This implies that the FET may be not as efficacious in expanding the true lumen in the chronic phase as in the acute phase, when 
the intimal flap is more mobile and flexible (21). Therefore, the distal aorta of patients with a small true lumen should be carefully evaluated preoperatively and preemptive extraanatomic bypass and endovascular stenting of distal aorta be considered beforehand to minimize the surgical risks $(7,30)$.

The natural history of cTAAD is dependent on several factors, such as patient age, the baseline aortic size, extent of dissection, false lumen patency, presence of a persistent communication, growth rate and control of hypertension $(3,31)$. In patients with connective tissue disorders, degeneration of the medial layer weakens the aortic wall and predisposes the aorta to dilatation, dissection and rupture. In this study, although overall survival did not differ significantly between patients operated on during the subacute (15-90 days) and chronic phases (after 90 days), the time from onset of symptoms to surgery was identified as a risk factor of late death. Given the limited efficacy of FET for true lumen expansion as shown in this cohort and the cumulative risk of adverse aortic events over time in young patients (3), we recommend that cTAAD should be repaired using the TAR and FET technique as early as possible, even at smaller aortic sizes in patients with MFS.

The study is limited by its retrospective nature, small sample size and the lack of a control group. With a larger number of patients, the difference in overall survival may become significant between the subacute and chronic groups. The extended period before surgery represents a selection bias occurring before referral to our team, whereby the less severe and complicated cases were more likely to have been managed by other hospitals, while the more severe and complicated cases to be referred and to reach our institution.

\section{Conclusions}

The TAR and FET technique is a safe and efficacious approach to cTAAD in patients with MFS, which achieved favorable early and late survival, and freedom from reoperation and dilatation. Given that the preoperative aortic size and duration from onset to surgery are predictive of early and long-term adverse events, earlier repair of cTAAD using the FET and TAR is recommended for such patients, even at smaller aortic sizes.

\section{Acknowledgments}

Funding: This work was supported by Beijing Major Science and Technology Projects (Z171100001017083
\& Z19110700660000) and the National Science and Technology Support Program of China (2015BAI12B03).

\section{Footnote}

Conflicts of Interest: The authors have no conflicts of interest to declare.

Open Access Statement: This is an Open Access article distributed in accordance with the Creative Commons Attribution-NonCommercial-NoDerivs 4.0 International License (CC BY-NC-ND 4.0), which permits the noncommercial replication and distribution of the article with the strict proviso that no changes or edits are made and the original work is properly cited (including links to both the formal publication through the relevant DOI and the license). See: https://creativecommons.org/licenses/by-nc-nd/4.0/.

\section{References}

1. Erbel R, Aboyans V, Boileau C, et al. 2014 ESC Guidelines on the diagnosis and treatment of aortic diseases. Eur Heart J 2014;35:2873-926.

2. Peterss S, Mansour AM, Ross JA, et al. Changing pathology of the thoracic aorta from acute to chronic dissection: Literature review and insights. J Am Coll Cardiol 2016;68:1054-65.

3. Kim WK, Park SJ, Kim HJ, et al. The fate of unrepaired chronic type A aortic dissection. J Thorac Cardiovasc Surg 2019;158:996-1004.e3.

4. Elefteriades JA. Natural history of thoracic aortic aneurysms: indications for surgery, and surgical versus nonsurgical risks. Ann Thorac Surg 2002;74:S1877-80; discussion S1892-8.

5. Rylski B, Milewski RK, Bavaria JE, et al. Outcomes of surgery for chronic type A aortic dissection. Ann Thorac Surg 2015;99:88-93.

6. Roselli EE, Bakaeen FG, Johnston DR, et al. Role of the frozen elephant trunk procedure for chronic aortic dissection. Eur J Cardiothorac Surg 2017;51:i35-9.

7. Ma WG, Zhang W, Zhu JM, et al. Long-term outcomes of frozen elephant trunk for type A aortic dissection in patients with Marfan syndrome. J Thorac Cardiovasc Surg 2017;154:1175-89.e2.

8. Sun LZ, Qi RD, Chang Q, et al. Surgery for Marfan patients with acute type A dissection using a stented elephant trunk procedure. Ann Thorac Surg 2008;86:1821-5. 
9. Sun LZ, Li M, Zhu JM, et al. Surgery for patients with Marfan syndrome with type A dissection involving the aortic arch using total arch replacement combined with stented elephant trunk implantation: The acute versus the chronic. J Thorac Cardiovasc Surg 2011;142:e85-91.

10. Chen Y, Ma WG, Zheng J, et al. Total arch replacement and frozen elephant trunk for type A aortic dissection after Bentall procedure in Marfan syndrome. J Thorac Dis 2018;10:2377-87.

11. Chen Y, Ma WG, Li JR, et al. Can frozen elephant trunk cure type I dissection confined to thoracic aorta in Marfan syndrome? Ann Thorac Surg 2020;109:1174-82.

12. Chen Y, Ma WG, Zhi AH, et al. Fate of distal aorta after frozen elephant trunk and total arch replacement for type A aortic dissection in Marfan syndrome. J Thorac Cardiovasc Surg 2019;157:835-49.

13. De Paepe A, Devereux RB, Dietz HC, et al. Revised diagnostic criteria for the Marfan syndrome. Am J Med Genet 1996;62:417-26.

14. Loeys BL, Dietz HC, Braverman AC, et al. The revised Ghent nosology for the Marfan syndrome. J Med Genet 2010;47:476-85.

15. Ge YP, Li CN, Chen L, et al. Is previous cardiac surgery a risk factor for short and mid-term mortality following total aortic arch replacement in patients with Stanford type A aortic dissection? Heart Lung Circ 2015;24:1111-7.

16. Ma WG, Zhu JM, Zheng J, et al. Sun's procedure for complex aortic arch repair: total arch replacement using a tetrafurcate graft with stented elephant trunk implantation. Ann Cardiothorac Surg 2013;2:642-8.

17. Zhong YL, Qi RD, Ma WG, et al. Frozen elephant trunk with modified en bloc arch reconstruction and left subclavian transposition for chronic type A dissection. J Thorac Dis 2018;10:5376-83.

18. Lin Y, Ma WG, Zheng J, et al. Supra-aortic vessel reconstruction in total arch replacement for acute type A dissection: Comparison of en bloc and separate graft techniques. Asian J Surg 2019;42:482-7.

19. Chou AS, Ma WG, Mok SC, et al. Do familial aortic dissections tend to occur at the same age? Ann Thorac Surg 2017;103:546-50.

20. Ma WG, Chou AS, Mok SCM, et al. Positive family history of aortic dissection dramatically increases dissection risk in family members. Int J Cardiol 2017;240:132-7.

21. Li Q, Ma WG, Zheng J, et al. Distal stent graft-induced new entry after TEVAR of type B aortic dissection: Experience in 15 years. Ann Thorac Surg 2019;107:718-24. 22. Li Q, Wang LF, Ma WG, et al. Risk factors for distal stent graft-induced new entry following endovascular repair of type B aortic dissection. J Thorac Dis 2015;7:1907-16.

23. Blackstone EH, Naftel DC, Turner ME. The decomposition of time-varying hazard into phases, each incorporating a separate stream of concomitant information. J Am Stat Assoc 1986;81:615-24.

24. Raghupathy A, Nienaber CA, Harris KM, et al. Geographic differences in clinical presentation, treatment, and outcomes in type A acute aortic dissection (from the International Registry of Acute Aortic Dissection). Am J Cardiol 2008;102:1562-6.

25. Harris KM, Strauss CE, Eagle KA, et al. Correlates of delayed recognition and treatment of acute type A aortic dissection: the International Registry of Acute Aortic Dissection (IRAD). Circulation 2011;124:1911-8.

26. Svensson LG, Crawford ES. Aortic dissection and aortic aneurysm surgery: clinical observations, experimental investigations, and statistical analyses. Part II. Curr Probl Surg 1992;29:913-1057.

27. Dagenais F. Commentary: Chronic type A dissection: When to operate? J Thorac Cardiovasc Surg 2019;158:1005-6.

28. Tsai TT, Isselbacher EM, Trimarchi S, et al. Acute type B aortic dissection: does aortic arch involvement affect management and outcomes? Insights from the International Registry of Acute Aortic Dissection (IRAD). Circulation 2007;116:I150-6.

29. Czerny M, Schmidli J, Adler S, et al. Current options and recommendations for the treatment of thoracic aortic pathologies involving the aortic arch: An expert consensus document of the European Association for CardioThoracic surgery (EACTS) and the European Society for Vascular Surgery (ESVS). Eur J Cardiothorac Surg 2019;5 5:133-62.

30. Pan XD, Li B, Ma WG, et al. Endovascular repair of residual intimal tear or distal new entry after frozen elephant trunk for type A aortic dissection. J Thorac Dis 2017;9:529-36.

31. Giugliano G, Spadera L, De Laurentis M, et al. Chronic aortic dissection: still a challenge. Acta Cardiol 2009;64:653-63.

Cite this article as: Chen Y, Ma WG, Li JR, Zheng J, Liu YM, Zhu JM, Sun LZ. Is the frozen elephant trunk technique justified for chronic type A aortic dissection in Marfan syndrome? Ann Cardiothorac Surg 2020;9(3):197-208. doi: 10.21037/acs.2020.03.10 